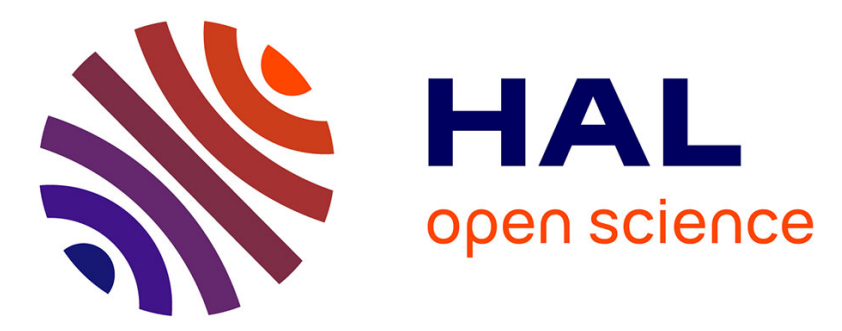

\title{
Axiomatising logics with separating conjunctions and modalities
}

\author{
Stephane Demri, Raul Fervari, Alessio Mansutti
}

\section{To cite this version:}

Stephane Demri, Raul Fervari, Alessio Mansutti. Axiomatising logics with separating conjunctions and modalities. 16th European Conference on Logics in Artificial Intelligence (JELIA'19), May 2019, Rende, Italy. 10.1007/978-3-030-19570-0_45 . hal-02362648

\section{HAL Id: hal-02362648 \\ https://hal.science/hal-02362648}

Submitted on 14 Nov 2019

HAL is a multi-disciplinary open access archive for the deposit and dissemination of scientific research documents, whether they are published or not. The documents may come from teaching and research institutions in France or abroad, or from public or private research centers.
L'archive ouverte pluridisciplinaire HAL, est destinée au dépôt et à la diffusion de documents scientifiques de niveau recherche, publiés ou non, émanant des établissements d'enseignement et de recherche français ou étrangers, des laboratoires publics ou privés. 


\title{
Axiomatising Logics with Separating Conjunction and Modalities
}

\author{
Stéphane Demri ${ }^{1}$, Raul Fervari ${ }^{2}$, and Alessio Mansutti ${ }^{1}$ \\ ${ }^{1}$ LSV, CNRS, ENS Paris-Saclay, Université Paris-Saclay, France \\ ${ }^{2}$ CONICET and Universidad Nacional de Córdoba, Argentina
}

\begin{abstract}
Modal separation logics are formalisms that combine modal operators to reason locally, with separating connectives that allow to perform global updates on the models. In this work, we design Hilbertstyle proof systems for the modal separation logics $\operatorname{MSL}(*,\langle\neq\rangle)$ and $\operatorname{MSL}(*, \diamond)$, where $*$ is the separating conjunction, $\diamond$ is the standard modal operator and $\langle\neq\rangle$ is the difference modality. The calculi only use the logical languages at hand (no external features such as labels) and take advantage of new normal forms and of their axiomatisation.
\end{abstract}

\section{Introduction}

Separation logics with epistemic flavour. Modal logic $[7,8]$ is a family of languages extending propositional logic with operators to describe and reason about different modes of truth. Such operators are usually called modalities. For instance, this family includes deontic (for permissions and obligations), epistemic (to reason about knowledge) and temporal modalities. On the other hand, separation logic [30,29] is a family of assertion languages originally conceived to perform Hoare-style verification [26] of programs with mutable data structures. The key components of separation logic are its non-classical connectives, that allow us to reason about updates of the models. For example, the formula $\phi * \psi$ uses the separating conjunction $*$, which requires to split a model into two disjoint pieces, one satisfying $\phi$ and the other one satisfying $\psi$. Over the last years, several approaches combining modal and separation logics have appeared. In most cases, the modal and the separation dimensions are orthogonal (see e.g. $[12,9,13]$ ), allowing us to design decision procedures by combinations of procedures from each dimension. However, recently, combinations of such operators interpreted over the same structures have been considered, see e.g. $[17,18]$. In this way, the underlying modal relational structure can be seen as a model from separation logic: states can be seen as memory locations, and edges can be seen as links between these locations.

These efforts on combining separation and modal logics witness the numerous attempts to use separation logic in different contexts. When interpreted on sets, separation logic can be used to model some particular phenomena in belief revision [25]. It can be combined with modalities from epistemic logic to capture reachable states (see the epistemic logic for resources introduced in [13]). Epistemic 
separation logic [15], where models have equivalence relations representing possible worlds, has been extended in [14] with public announcements. Lastly, in [28] operators from temporal and separation logics are combined, allowing to express both temporal and spatial conditions in search control knowledge for AI planning (see also [10]). From a logical perspective, modal operators to perform updates on a relational model can be seen as weaker versions or variants of separating connectives, since they all have similar effects: updating the model (by adding, removing or changing some feature of the model) while evaluating a formula. For example, consider the sabotage modal logic SML introduced in [34] (see [24] for application in formal learning theory). SML is an extension of the basic modal language with a so-called sabotage operator which deletes one arrow of the model when it is evaluated. This operator can be seen as a weak version of the separating conjunction that separates only one edge from the rest of the model (see [18] for details). Other examples of dynamic logics used to describe graph evolution in games can be found in $[33,3]$ (see also [16]).

Due to their ability to perform updates on a relational model, designing proof methods for such logics is known to be a non-trivial task. As a matter of fact, no proof system without features external from the logical language is known for the above-mentioned logics. For instance, there exist tableaux-based procedures to check satisfiability of sabotage logics $[2,4]$ but model updates are handled with labels. Moreover, the rules in these calculi are quite complex, and they are far from providing a good understanding of the logics. On the other hand, there are no Hilbert-style calculi, as it is extremely challenging to axiomatise these logics that do not satisfy the uniform substitution rule (see e.g. [3]).

Our motivations. We pursue a research program about modal separation logics to better understand the computational complexity of their decision problems and to design proof systems, such as Hilbert-style calculi. These calculi have clearly an historical value but also provide essential means to grasp what are the core validities and rules of the logical formalisms, see a recent illustration in [1]. It should be noted that not all modal separation logics admit finite axiomatisation, see e.g. [18], and sometimes, the axiomatisation of abstract separation logics requires the need for external features such as nominals or labels, see e.g. [11,27]. In this work, we adopt a puristic approach to design Hilbert-style proof systems for the very logical language without any external help. In the context of modal separation logics, this is a requirement that happens to be rewarding for understanding their expressive power, considering that such logics freely mix modal operators and separating connectives having global effects.

Our contribution. We design sound and complete Hilbert-style proof systems for the modal separation logics $\operatorname{MSL}(*, \diamond)$ and $\operatorname{MSL}(*,\langle\neq\rangle)[18]$, where $*$ is the separating conjunction, $\diamond$ is the standard modal operator and $\langle\neq\rangle$ is the difference modality. In both cases, we provide a syntactical treatment to the semantical abstractions used to decide such logics in [18], leading to NP-completeness. Each formula is shown equivalent to a Boolean combination of core formulae: simple formulae of the logic expressing elementary properties about the models. More precisely, each elementary property consists of a "modal part" (describing 
partially the structure of the model), and a "size part" (related to the number of edges). Thus, we show how to introduce axioms to transform every formula into a Boolean combination of core formulae, together with axioms to deal with these simple formulae. This result borrows some ideas from the Gaifman's Theorem in first-order logic [21], which states that every first-order sentence is logically equivalent to a Boolean combination of so-called local formulae. A similar strategy is also followed for axiomatising dynamic epistemic logics $[36,35,37]$ with the introduction of reduction axioms. In this technique, it is essential to translate each formula containing a dynamic operator into a formula without it, by using provably equivalent formulae. Then, completeness follows from the completeness of the system for the 'basic' language (see also a similar approach for the linear $\mu$ calculus in [20]). In our case, another difficulty arises as we also have to design an axiomatisation for such Boolean combinations. The proof system for $\operatorname{MSL}(*, \diamond)$ (§3) uses partially the standard machinery for modal logic, but it is a bit different from the axiomatisation for the modal logic $\mathrm{Alt}_{1}$, i.e., the modal logic over deterministic frames, characterised by the axiom $\diamond p \Rightarrow \square p$ (see e.g. [5]). For $\operatorname{MSL}(*,\langle\neq\rangle)(\S 4)$, the modal part extends results from [32] to infinite models (a peculiarity of modal separation logics as the set of locations is infinite). These constructions give us an exact characterisation of the properties that can be expressed on each logic. Moreover, it is also remarkable to have axiomatisations for these two NP-complete logics, since the full logic MSL (including the separating implication) is not (finitely) axiomatisable [18].

\section{Preliminaries about modal separation logics}

We briefly recall the definition of the modal separation logic $\operatorname{MSL}(*, \diamond,\langle\neq\rangle)$ introduced in [18]. Let $\mathrm{PROP}=\{p, q, \ldots\}$ be a countably infinite set of propositional symbols. Formulae of the $\operatorname{logic} \operatorname{MSL}(*, \diamond,\langle\neq\rangle)$ are defined by the grammar:

$$
\phi::=\top|p| \operatorname{emp}|\neg \phi| \phi \vee \phi|\diamond \phi|\langle\neq\rangle \phi \mid \phi * \phi,
$$

where $p \in \operatorname{PROP}$ (as usual $\perp \stackrel{\text { def }}{=} \neg \top$ ). A model is a tuple $\mathfrak{M}=\langle\mathbb{N}, \mathfrak{R}, \mathfrak{V}\rangle$ such that

- the set of locations is the set of natural numbers $\mathbb{N}$,

$-\mathfrak{R} \subseteq \mathbb{N} \times \mathbb{N}$ is finite and weakly functional (a.k.a. deterministic, i.e. $\left(\mathfrak{l}, \mathfrak{l}^{\prime}\right) \in \mathfrak{R}$ and $\left(\mathfrak{l}, \mathfrak{l}^{\prime \prime}\right) \in \mathfrak{R}$ imply $\left.\mathfrak{l}^{\prime}=\mathfrak{l}^{\prime \prime}\right)$ and,

$-\mathfrak{V}: \operatorname{PROP} \rightarrow \mathcal{P}(\mathbb{N})$ is a valuation.

In the rest of the document, by 'functional', we mean 'weakly functional'. Since separation logics are interpreted on structures representing heaps [6], this explains why in the models, the domain is $\mathbb{N}$ (an infinite set of locations), and the accessibility relation is finite and functional (formal relationships with separation logics can be found in $[18, \S 2.2])$. The models $\mathfrak{M}_{1}=\left\langle\mathbb{N}, \mathfrak{R}_{1}, \mathfrak{V}\right\rangle$ and $\mathfrak{M}_{2}=$ $\left\langle\mathbb{N}, \mathfrak{R}_{2}, \mathfrak{V}\right\rangle$ are disjoint if $\mathfrak{R}_{1} \cap \mathfrak{R}_{2}=\emptyset$; when this holds, $\mathfrak{M}_{1} \uplus \mathfrak{M}_{2}$ denotes the model corresponding to the disjoint union of $\mathfrak{M}_{1}$ and $\mathfrak{M}_{2}$. Given $\mathfrak{M}=\langle\mathbb{N}, \mathfrak{R}, \mathfrak{V}\rangle$ 
and $\mathfrak{l} \in \mathbb{N}$, the satisfaction relation $\models$ is defined below (we omit standard clauses for Boolean connectives):

$$
\begin{array}{lll}
\mathfrak{M}, \mathfrak{l}=p & \stackrel{\text { def }}{\Leftrightarrow} & \mathfrak{l} \in \mathfrak{V}(p) \quad \mathfrak{M}, \mathfrak{l}=\text { emp } \stackrel{\text { def }}{\Leftrightarrow} \mathfrak{R}=\emptyset \\
\mathfrak{M}, \mathfrak{l}=\diamond \phi & \stackrel{\text { def }}{\Leftrightarrow} & \mathfrak{M}, \mathfrak{l}^{\prime}=\phi, \text { for some } \mathfrak{l}^{\prime} \in \mathbb{N} \text { such that }\left(\mathfrak{l}, \mathfrak{l}^{\prime}\right) \in \mathfrak{R} \\
\mathfrak{M}, \mathfrak{l}=\langle\neq\rangle \phi & \stackrel{\text { def }}{\Leftrightarrow} & \mathfrak{M}, \mathfrak{l}^{\prime}=\phi, \text { for some } \mathfrak{l}^{\prime} \in \mathbb{N} \text { such that } \mathfrak{l}^{\prime} \neq \mathfrak{l} \\
\mathfrak{M}, \mathfrak{l}=\phi_{1} * \phi_{2} & \stackrel{\text { def }}{\Leftrightarrow} & \left\langle\mathbb{N}, \mathfrak{R}_{1}, \mathfrak{V}\right\rangle, \mathfrak{l}=\phi_{1} \text { and }\langle\mathbb{N}, \mathfrak{R}, \mathfrak{V}\rangle, \mathfrak{l} \models \phi_{2}, \\
& & \text { for some partition }\left\{\mathfrak{R}_{1}, \mathfrak{R}_{2}\right\} \text { of } \mathfrak{R} .
\end{array}
$$

The semantics for the modal operators and the separating connectives is the standard one, see e.g. [7,30]. The restriction of MSL $(*, \diamond,\langle\neq\rangle)$ without the modal operator $\diamond($ resp. $\langle\neq\rangle)$ is denoted by $\operatorname{MSL}(*,\langle\neq\rangle)($ resp. $\operatorname{MSL}(*, \diamond))$. It is established in [18] that the satisfiability problems for MSL $(*, \diamond)$ and $\operatorname{MSL}(*,\langle\neq\rangle)$ are NP-complete whereas the problem for $\operatorname{MSL}(*,\langle\neq\rangle, \diamond)$ is ToweR-complete ${ }^{1}$.

To illustrate the expressive power of $\operatorname{MSL}(*, \diamond)$, let us define loop $_{1}$, which states that the model consists of a single reflexive edge at the evaluation point:

$$
\neg \operatorname{emp} \wedge \neg(\neg \text { emp } * \neg \text { emp }) \wedge \diamond \diamond \top .
$$

Moreover, it is possible to define the formula $\mathrm{loop}_{2}$, that interpreted on a location $\mathfrak{l}$, states that the model contains exactly a loop of length 2 visiting $\mathfrak{l}$ :

$$
\begin{gathered}
(\neg \text { emp } * \neg \text { emp }) \wedge \neg(\neg \text { emp } * \neg \text { emp } * \neg \text { emp }) \wedge \diamond \diamond \diamond \top \wedge \\
\neg(\neg \text { emp } * \diamond \diamond \diamond \top) \wedge \neg \diamond(\neg \text { emp } * \diamond \diamond \diamond \top) .
\end{gathered}
$$

Notice that $*$ is associative. Obviously, these properties cannot be expressed in the modal logic Alt $_{1}$.

So, in this paper, we aim at providing Hilbert-style axiomatisations for $\operatorname{MSL}(*, \diamond)$ and $\operatorname{MSL}(*,\langle\neq\rangle)$, which amounts to characterise syntactically the set of valid formulae by means of a proof system. By contrast, the complexity results from [18] are obtained semantically, without any proof-theoretical analysis.

\section{Axiomatising $\operatorname{MSL}(*, \diamond)$ with core formulae}

In this section, we define a proof system for $\operatorname{MSL}(*, \diamond)$, namely $\mathcal{H} \operatorname{MSL}(*, \diamond)$. To do so, we introduce a set of core formulae that are simple formulae capturing essential properties. As shown later on, every $\operatorname{MSL}(*, \diamond)$ formula is logically equivalent to a Boolean combination of core formulae. However, as every core formula is shown to be an $\operatorname{MSL}(*, \diamond)$ formula, we can derive an axiomatisation of $\operatorname{MSL}(*, \diamond)$ by axiomatising Boolean combinations of core formulae. So, we define three sets of axioms and inference rules: (1) those dedicated to the propositional logic of core formulae, (2) those that, given a Boolean combination of core formulae $\phi$, allow to derive a Boolean combination of core formulae that is equivalent to $\diamond \phi$ (a property called herein $\diamond$-elimination, see Lemma 6 ), and

\footnotetext{
${ }^{1}$ The class Tower is the class of problems of time complexity bounded by a tower of exponentials, whose height is an elementary function of the input. See [31] for details.
} 
(3) those that, given two Boolean combinations of core formulae $\phi_{1}, \phi_{2}$, allow to derive a Boolean combination of core formulae that is equivalent to $\phi_{1} * \phi_{2}$ (a property called herein $*$-elimination, see Lemma 9).

Core formulae for $\operatorname{MSL}(*, \diamond)$. Core formulae are divided into two families: a set of size formulae that express properties about the size of the model (i.e. the number of edges) and a set of graph formulae describing the shape of the model that is observable from the current location. As the relation $\mathfrak{R}$ in models is weakly functional, the number of distinct shapes is limited, ranging from lasso shapes to segments with dead-end.

Let us introduce expressions of the form size $\geq \beta$ that hold true whenever $\mathfrak{R}$ has at least $\beta$ elements (the symbol $\beta$ always refers to a natural number throughout the paper). A size literal is a formula of the form size $\geq \beta$ or $\neg$ size $\geq \beta$. Every Boolean combination of size literals is a size formula. We also use size $=\beta$ as an abbreviation for size $\geq \beta \wedge \neg$ size $\geq \beta+1$. At this stage, it is worth noting that size $\geq \beta$ should be understood as a built-in atomic formula enriching the logical language for $\operatorname{MSL}(*, \diamond)$. However, as it will quickly appear below, size $\geq \beta$ can be characterised with a formula of $\operatorname{MSL}(*, \diamond)$ and later on in the document, such occurrences of size $\geq \beta$ should be understood as mere abbreviations. The same distinction applies to the graph formulae defined below.

Graph formulae describe the shape of a portion of the model, partly inspired from the semantical notion of abstract frame from $[18, \S 4.1]$ but with constraints on propositional variables. Formally, every graph formula is an expression derived from the non-terminal $\mathcal{G}$ of the grammar below:

$$
\ell:=\top|\perp| p|\neg p \quad Q:=\ell| Q \wedge Q \quad \mathcal{G}:=|Q, \ldots, Q\rangle|| Q, \ldots, Q]|| Q, \ldots, \overleftarrow{Q, \ldots, Q} \mid
$$

where $p \in \mathrm{PROP}$, and $\mathcal{G}$ must contain at least one conjunction $Q$. By slightly abusing the standard terminology, expressions of the form $\ell$ are called literals. A conjunction $Q$ is contradictory whenever $\perp$ occurs in $Q$ or there is some $p$ such that both $p$ and $\neg p$ occur in $Q$. Note that $Q$ is contradictory iff $Q$ is unsatisfiable. By convention, contradictory conjunctions are denoted by $Q^{\perp}$. A graph formula is contradictory if at least one of its conjunctions is contradictory. Note also that the semantics for graph formulae shall guarantee that a graph formula is contradictory iff it is unsatisfiable.

Since we are working on weakly functional and finite relations, graph formulae represent paths satisfying a conjunction of literals $Q$ at each position. A formula of the form $\left|Q_{1}, \ldots, Q_{n}\right\rangle$ expresses that there exists a path of length $n$ in which all the locations are distinct of each other, and we do not know whether it continues after. The formula $\left.\mid Q_{1}, \ldots, Q_{n}\right]$ states that there is a path of length $n-1$, all the locations are distinct, and the last location has no successor. Finally, the formula of the form $\left|Q_{1}, \ldots, \overleftarrow{Q_{i}, \ldots, Q_{n}}\right|$ expresses that there is a path of size $n-1$ with all distinct locations, and there is a loop from the location in position $n$ and the one in the position $i$ (lasso shape). Sometimes, we write $\mid Q_{1}, \ldots, Q_{n}$ ? to refer to graph formulae of any kind. Furthermore, we write $\mid Q, \ldots, Q^{\prime} ?_{(n)}$ to express that the last argument $Q^{\prime}$ of the corresponding graph formula is at position $n$. For example, 
$\mid \top, \ldots, \top]_{(5)}$ stands for $\left.\mid \top, \top, \top, \top, \top\right]$. Lastly, we write $\sharp\left(\mid Q_{1}, \ldots, Q_{n}\right.$ ? $)$ to denote the graph size of $\mid Q_{1}, \ldots, Q_{n}$ ? defined as follows:

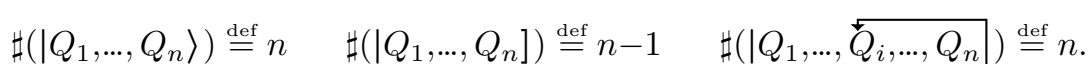

Given $\mathfrak{M}=\langle\mathbb{N}, \mathfrak{R}, \mathfrak{V}\rangle$ and $\mathfrak{l} \in \mathbb{N}$, the relation $\models$ is extended to core formulae:

$$
\begin{aligned}
& \mathfrak{M}, \mathfrak{l}=\operatorname{size} \geq \beta \quad \stackrel{\text { def }}{\Leftrightarrow} \operatorname{card}(\mathfrak{R}) \geq \beta \\
& \mathfrak{M}, \mathfrak{l}=\left|Q_{1}, \ldots, Q_{n}\right\rangle \quad \stackrel{\text { def }}{\Leftrightarrow} \text { there are distinct } \mathfrak{l}_{1}, \ldots, \mathfrak{l}_{n+1} \text { s.t. } \mathfrak{l}=\mathfrak{l}_{1} \mathfrak{R} \mathfrak{l}_{2} \mathfrak{R} \ldots \mathfrak{R} \mathfrak{l}_{n+1} \text {, } \\
& \text { and for all } j \in[1, n], \mathfrak{M}, \mathfrak{l}_{j}=Q_{j} \\
& \left.\mathfrak{M}, \mathfrak{l}=\mid Q_{1}, \ldots, Q_{n}\right] \quad \stackrel{\text { def }}{\Leftrightarrow} \text { there are distinct } \mathfrak{l}_{1}, \ldots, \mathfrak{l}_{n} \text { s.t. } \mathfrak{l}=\mathfrak{l}_{1} \mathfrak{R l} \mathfrak{l}_{2} \mathfrak{R} \ldots \mathfrak{R} \mathfrak{l}_{n}, \\
& \mathfrak{R}\left(\mathfrak{l}_{n}\right)=\emptyset \text { and for each } j \in[1, n], \mathfrak{M}, \mathfrak{l}_{j}=Q_{j} \\
& \mathfrak{M}, \mathfrak{l}=\left|Q_{1}, \ldots, \overleftarrow{Q_{i}, \ldots, Q_{n}}\right| \stackrel{\text { def }}{\Leftrightarrow} \text { there are distinct } \mathfrak{l}_{1}, \ldots, \mathfrak{l}_{n} \text { s.t. } \mathfrak{l}=\mathfrak{l}_{1} \mathfrak{R} \mathfrak{l}_{2} \mathfrak{R} \ldots \mathfrak{l}_{n} \mathfrak{R} \mathfrak{R l}_{i} \\
& \text { and for all } j \in[1, n], \mathfrak{M}, \mathfrak{l}_{j}=Q_{j} \text {. }
\end{aligned}
$$

Below, we establish that every core formula has a logically equivalent counterpart in $\operatorname{MSL}(*, \diamond)$ (Lemma 1). This is an essential property as these formulae are the building blocks of the axiomatisation of $\operatorname{MSL}(*, \diamond)$. Consequently, we obtain that our axioms are only made of $\operatorname{MSL}(*, \diamond)$ formulae, with no need for external properties or extra machinery such as nominals or labels.

For every core formula $\psi$, we define its extension $\operatorname{ext}(\psi)$ in $\operatorname{MSL}(*, \diamond)$.

$$
\begin{aligned}
& -\operatorname{ext}(\text { size } \geq 0) \stackrel{\text { def }}{=} T \text { and } \operatorname{ext}(\text { size } \geq \beta) \stackrel{\text { def }}{=} \overbrace{\neg \operatorname{emp} * \cdots * \neg \text { emp }}^{\beta \text { times }} \beta>0 \text {. } \\
& \left.\left.-\operatorname{ext}(\mid Q]) \stackrel{\text { def }}{=} Q \wedge \neg \diamond \top \text {. For } n \geq 2, \operatorname{ext}\left(\mid Q_{1}, Q_{2}, \ldots, Q_{n}\right]\right) \stackrel{\text { def }}{=} Q_{1} \wedge \diamond \operatorname{ext}\left(\mid Q_{2}, \ldots, Q_{n}\right]\right) \text {. } \\
& \left.-\operatorname{ext}\left(\left|Q_{1}, \ldots, Q_{n}\right\rangle\right) \stackrel{\text { def }}{=} \operatorname{ext}\left(\mid Q_{1}, \ldots, Q_{n}, \top\right]\right) * \top \text {. } \\
& -\operatorname{ext}\left(\left|\overleftarrow{Q_{1}, \ldots, Q_{n}}\right|\right) \text { is defined as the formula } \\
& \left.\top *\left(\operatorname{ext}(\operatorname{size}=n) \wedge \diamond^{n+1} \top \wedge\left(\operatorname{ext}\left(\mid Q_{1}, \ldots, Q_{n}\right]\right) * \top\right) \wedge \neg \diamond\left(\operatorname{ext}(\operatorname{size}=1) * \diamond^{n} \top\right)\right) \\
& \text { where } \diamond^{0} \phi \stackrel{\text { def }}{=} \phi \text { and } \diamond^{i+1} \phi \stackrel{\text { def }}{=} \diamond \diamond^{i} \phi \text {. For } i>1 \text {, ext }\left(\left|Q_{1}, \ldots, \overleftarrow{Q_{i}, \ldots, Q_{n} \mid}\right|\right) \text { is } \\
& \begin{array}{r}
\top *\left(\operatorname{ext}(\operatorname{size}=n) \wedge \diamond^{n+1} \top \wedge\left(\operatorname{ext}\left(\mid Q_{1}, \ldots, Q_{n}\right]\right) * \top\right) \wedge \\
\left.\diamond^{i-1}\left(\operatorname{ext}(\operatorname{size}=i-1) * \operatorname{ext}\left(||^{\dagger}, \ldots,\left.\top\right|_{(n-i+1)}\right)\right)\right) .
\end{array}
\end{aligned}
$$

Lemma 1. All the core formulae $\psi$ are logically equivalent to $\operatorname{ext}(\psi)$.

From now on, for any occurrence of a core formula $\psi$, including occurrences in the axioms or inference rules, we mean the formula ext $(\psi)$ so that their provisory status of built-in atomic formula is upgraded to a permanent abbreviation.

Hilbert-style proof system for $\operatorname{MSL}(*, \diamond)$. To obtain an axiomatisation of $\operatorname{MSL}(*, \diamond)$, we start by introducing the proof system $\mathcal{H}_{c}$ dedicated to Boolean combinations of core formulae. As $\operatorname{MSL}(*, \diamond)$ includes the propositional logic, $\mathcal{H}_{c}$ and all the subsequent proof systems contain the axiom schemas and modus ponens for the propositional calculus. Throughout the paper we use standard notations about Hilbert-style proof systems. To simplify, sometimes we will abuse the terminology and use 'axiom' instead of 'axiom schema'. The axioms whose name is of the form $\mathbf{G}_{i}^{\text {? }}$ (resp. $\mathbf{S}_{i}^{?}$ ) handle graph formulae (resp. size formulae). 
We start with the axioms for size $\geq \beta$, its interactions with graph formulae and one axiom schema for inconsistent graph formulae.

Axioms for size formulae and for inconsistent graph formulae

\begin{tabular}{ll}
\hline $\left.\mathbf{S}_{1}^{c}\right)$ size $\geq 0$ & $\left(\mathbf{G}_{1}^{c}\right) \mid Q_{1}, \ldots, Q_{n} ? \Rightarrow$ size $\geq \sharp\left(\mid Q_{1}, \ldots, Q_{n} ?\right)$ \\
$\left(\mathbf{S}_{2}^{c}\right)$ size $\geq \beta+1 \Rightarrow$ size $\geq \beta$ & $\left(\mathbf{G}_{2}^{c}\right) \neg \mid \ldots, Q^{\perp}, \ldots ?$ \\
\hline
\end{tabular}

The meaning of these axioms is straightforward. For instance, the axiom $\left(\mathbf{S}_{2}^{c}\right)$ states that if the accessibility relation of a model has at least $\beta+1$ elements, then it has at least $\beta$ elements. The axiom $\left(\mathbf{G}_{1}^{c}\right)$ states that if a model satisfies a graph formula $\mathcal{G}$ then its accessibility relation cannot have less elements than its graph size. We complete the definition of $\mathcal{H}_{c}$ with two families of axioms, involving graph formulae. The first family (with the axioms from $\left(\mathbf{G}_{3}^{c}\right)$ to $\left(\mathbf{G}_{13}^{c}\right)$ ) concerns conjunctions of graph formulae. In particular, given two graph formulae, these axioms allow to derive an equivalent graph formula. Similarly, the second family (with the axioms from $\left(\mathbf{G}_{14}^{c}\right)$ to $\left(\mathbf{G}_{16}^{c}\right)$ ) concerns the negation of a graph formula. With these axioms, every negation of a graph formula is shown equivalent to a disjunction of graph formulae. Let us begin with the first family.

Axioms for conjunction of graph formulae

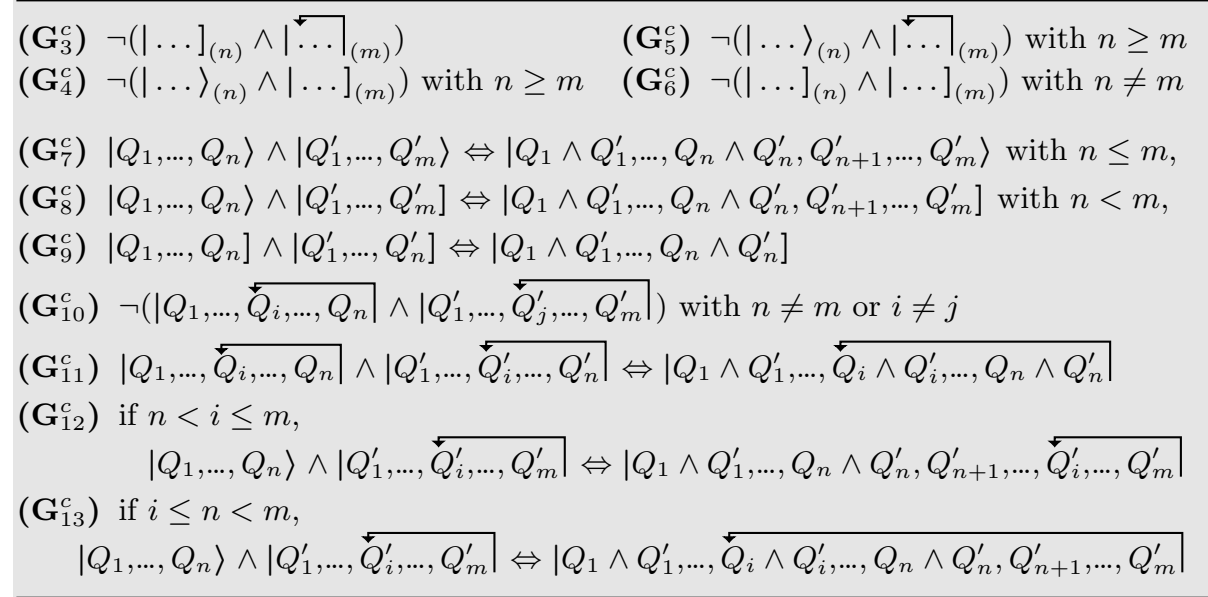

Thanks to these axioms, any conjunction of two graph formulae is valid only if it express properties that can be found together in a single model. For instance, $\mid \ldots]\left._{(n)} \wedge\right|_{(m)}$ is clearly contradictory (see the axiom $\left(\mathbf{G}_{3}^{c}\right)$ ), as the existence of a loop contradicts the fact that there is a dead-end (i.e. a location without successors). To ease the readability of the axioms for negation, we first define some auxiliary formulae.

$\left.\rho_{n} \stackrel{\text { def }}{=}|\top, \ldots, \top\rangle_{(n)} \quad \tau_{n} \stackrel{\text { def }}{=} \bigvee_{i \in[1, n]} \mid \top, \ldots, \top\right]_{(i)} \quad \lambda_{n} \stackrel{\text { def }}{=} \bigvee_{\substack{i \in[1, n] \\ j \in[1, i]}} \mid \top, \ldots, \underset{j}{\stackrel{\digamma}{\dagger}, \ldots,\left.\top\right|_{(i)}}$ In $\lambda_{n}$, the index $j$ below $\top$ indicates that the loop begins at the $j$-th position. We introduce the involution $\overline{(.)}$ on literals so that for every $p \in \operatorname{PROP}, \bar{p} \stackrel{\text { def }}{=} \neg p$, $\overline{\neg p} \stackrel{\text { def }}{=} p, \bar{\top} \stackrel{\text { def }}{=} \perp$ and $\perp \stackrel{\text { def }}{=} T$. This development is needed since graph formulae do not admit doubly negated literals. We write $\ell \in Q$ to denote that $\ell$ is a literal 
occurring in $Q$ with the same polarity. So, $\neg p$ appearing in $Q$ does not imply $p \in Q$. The axioms for dealing with negation are defined as follows.

Axioms for negation of graph formulae

$$
\begin{aligned}
& \left(\mathbf{G}_{14}^{c}\right) \neg\left|Q_{1}, \ldots, Q_{n}\right\rangle \Leftrightarrow \lambda_{n} \vee \tau_{n} \vee \bigvee_{\substack{i \in[1, n] \\
\ell \in Q_{i}}}\left|\top, \ldots, \bar{\ell}_{i}, \ldots, T\right\rangle_{(n)} \\
& \left.\left.\left(\mathbf{G}_{15}^{c}\right) \neg \mid Q_{1}, \ldots, Q_{n}\right] \Leftrightarrow \rho_{n} \vee \tau_{n-1} \vee \lambda_{n} \vee \bigvee_{\substack{i \in[1, n] \\
\ell \in Q_{i}}} \mid \top, \ldots, \bar{\ell}, \ldots, \top\right]_{(n)} \\
& \left(\mathbf{G}_{16}^{c}\right) \neg\left|Q_{1}, \ldots, \overleftarrow{Q_{i}, \ldots, Q_{n}}\right| \Leftrightarrow \rho_{n} \vee \tau_{n} \vee \lambda_{n-1} \vee \bigvee_{i \in[1, n-1]}\left|\top, \ldots, \bar{\ell}_{i} \ldots, \top\right\rangle_{(n-1)}
\end{aligned}
$$

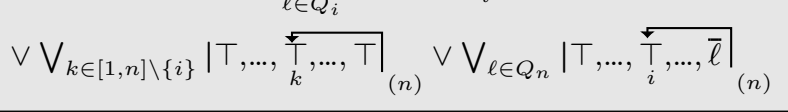

These axioms characterise the shape of the accessibility relation when one particular shape is excluded. For example, if $\mathfrak{M}, \mathfrak{l} \models \neg \mid \top, \top, \top]$, then the path starting from $\mathfrak{l}$ is of length 0,1 or greater than 2 . When it is of length 2 (equal to $\sharp(\mid \top, T, \top]))$, it has a lasso shape. These cases are captured by the axiom $\left(\mathbf{G}_{15}^{c}\right)$.

Lemma 2. Every axiom in $\mathcal{H}_{c}$ is valid for $\operatorname{MSL}(*, \diamond)$.

To show the completeness of $\mathcal{H}_{c}$, we exploit its ability to eliminate negations and conjunctions of graph formulae. This is enough to show that every Boolean combination of core formulae is equivalent to a disjunction of formulae of the form either $\mathcal{G} \wedge$ size $\geq \beta$ or $\mathcal{G} \wedge$ size $\geq \beta \wedge \neg$ size $\geq \beta^{\prime}$, where $\mathcal{G}$ is a graph formula. Such formulae are called elementary shapes.

Lemma 3. Let $\phi$ be a Boolean combination of core formulae. There is a disjunction of elementary shapes $\psi$ such that $\vdash_{\mathcal{H}_{c}} \phi \Leftrightarrow \psi$.

By Lemma 2, the formulae $\phi$ and $\psi$ in Lemma 3 are logically equivalent. We prove that $\mathcal{H}_{c}$ is complete for the restricted case of elementary shapes.

Lemma 4. Let $\phi$ be an elementary shape. $\phi$ is satisfiable iff $\nvdash_{\mathcal{H}_{c}} \neg \phi$.

From this result, we can establish the completeness of $\mathcal{H}_{c}$ with respect to Boolean combinations of core formulae. This is an essential step to get a complete proof system for $\operatorname{MSL}(*, \diamond)$ (its definition is to be completed in the rest of $\S 3$ ).

Theorem 1. A Boolean combination of core formulae $\phi$ is valid iff $\vdash_{\mathcal{H}_{c}} \phi$.

Proof. Let $\phi$ be a Boolean combination of core formulae. By Lemma $2, \vdash_{\mathcal{H}_{c}} \phi$ implies that $\phi$ is valid. Conversely, let us assume that $\phi$ is valid and ad absurdum, let us suppose that $\nvdash_{\mathcal{H}_{c}} \phi$. By propositional calculus, there exists a formula $\phi^{\prime}$ in conjunctive normal form (CNF) such that the "literals" are core formulae or their negations, and $\vdash_{\mathcal{H}_{c}} \phi \Leftrightarrow \phi^{\prime}$. As $\forall_{\mathcal{H}_{c}} \phi$, there is a conjunct of $\phi^{\prime}$, say $\psi$, such that $\nvdash_{\mathcal{H}_{c}} \psi$. As $\phi^{\prime}$ is valid, this implies that $\psi$ is valid too. By Lemma 3, $\vdash \mathcal{H}_{c} \neg \psi \Leftrightarrow\left(\varphi_{1} \vee \cdots \vee \varphi_{n}\right)$ where $\varphi_{1} \vee \cdots \vee \varphi_{n}$ is a disjunction of elementary shapes and therefore $\left(\varphi_{1} \vee \cdots \vee \varphi_{n}\right)$ is unsatisfiable. Consequently, for all $i \in[1, n]$, the formula $\varphi_{i}$ is unsatisfiable and by Lemma 4 , we get that $\vdash_{\mathcal{H}_{c}} \neg \varphi_{i}$. By propositional reasoning, we get $\vdash_{\mathcal{H}_{c}} \neg \varphi_{1} \wedge \cdots \wedge \neg \varphi_{n}$ and again by propositional reasoning using $\vdash_{\mathcal{H}_{c}} \neg \psi \Leftrightarrow\left(\varphi_{1} \vee \cdots \vee \varphi_{n}\right)$, we obtain $\vdash_{\mathcal{H}_{c}} \psi$, which leads to a contradiction. 
$\diamond$-elimination. We enrich $\mathcal{H}_{c}$ by adding axioms and one inference rule that handle $\diamond$, leading to the extended proof system $\mathcal{H}_{c}(\diamond)$ dedicated to the set of formulae obtained by closing core formulae under Boolean connectives and $\diamond$.

Axioms and inference rule for $\mathcal{H}_{c}(\diamond)$

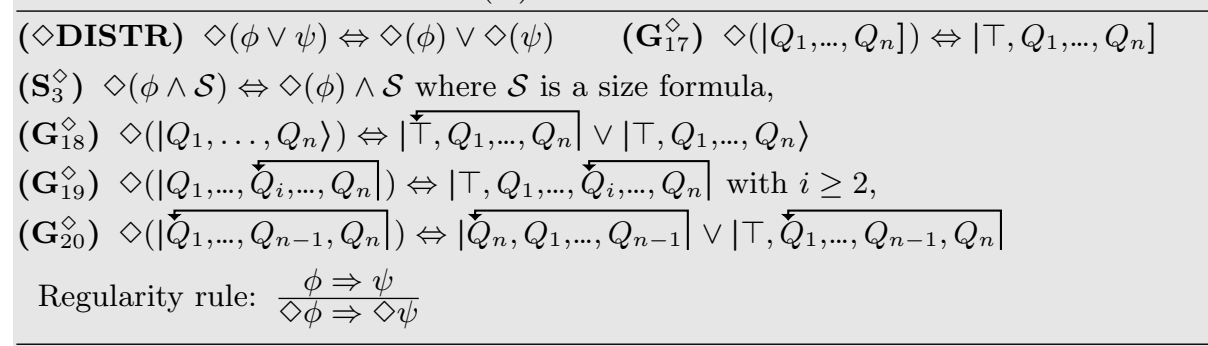

Lemma 5. Axioms and rules in $\mathcal{H}_{c}(\diamond)$ are valid for $M S L(*, \diamond)$.

These axioms give us some insight about the interplay between separating and modal connectives. In the case of size formulae there is no interplay at all (see the axiom $\left(\mathbf{S}_{3}^{\diamond}\right)$ ). Indeed, every condition in a formula $\psi$ about the size of the accessibility relation $\mathfrak{R}$ carries on independently of the structure of $\mathfrak{R}$ described by $\psi$ through modalities. However, there are interplays with respect to loops (see e.g. the axiom $\left(\mathbf{G}_{18}^{\diamond}\right)$ and recall that $\operatorname{ext}\left(\left|Q_{1}, \ldots, \overleftarrow{Q_{i}, \ldots, Q_{n}}\right|\right)$ uses $\left.*\right)$.

Lemma 6. Let $\phi$ be a Boolean combination of core formulae. There is a Boolean combination of core formulae $\psi$ such that $\vdash_{\mathcal{H}_{c}(\diamond)} \diamond \phi \Leftrightarrow \psi$.

By Lemma 5 , the formulae $\diamond \phi$ and $\psi$ in Lemma 6 are logically equivalent.

*-elimination. Finally, we enrich $\mathcal{H}_{c}$ by adding axioms and one inference rule for the separating conjunction. We denote the resulting proof system by $\mathcal{H}_{c}(*)$. Axioms and inference rule for $\mathcal{H}_{c}(*)$

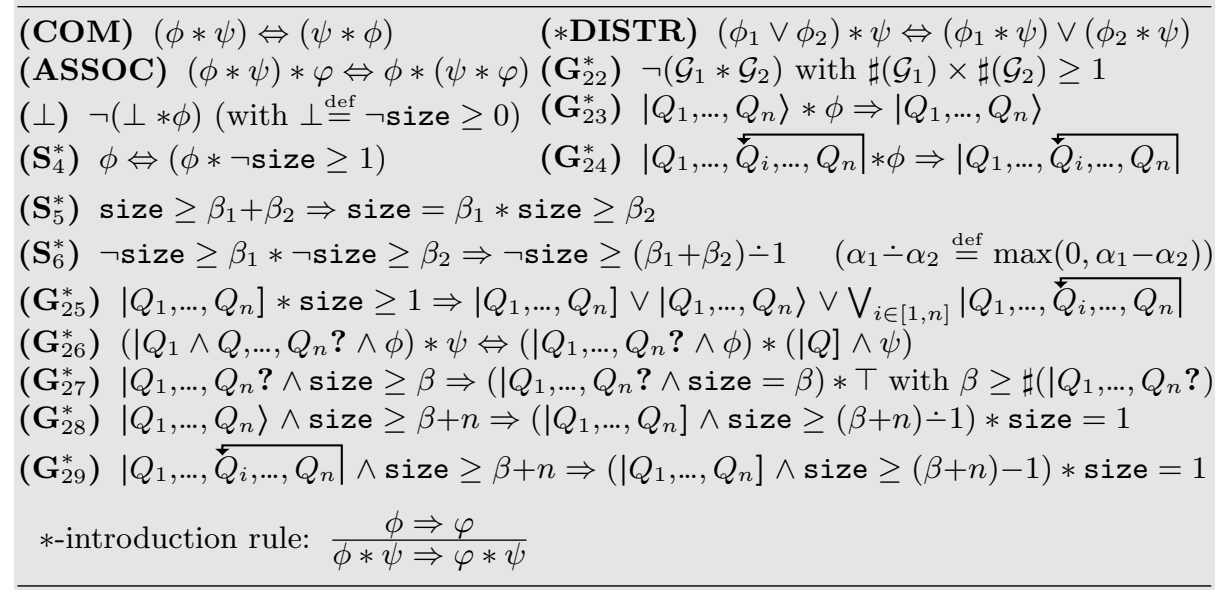

The first property to check is the soundness of $\mathcal{H}_{c}(*)$. 
Lemma 7. Axioms and rules in $\mathcal{H}_{c}(*)$ are valid for $M S L(*, \diamond)$.

Forthcoming Lemma 9 states that the separating conjunction $\phi * \psi$ of two Boolean combinations of core formulae is equivalent in $\mathcal{H}_{c}(*)$ to some Boolean combination of core formulae $\varphi$, and therefore by Lemma $7, \phi * \psi$ is also $\log$ ically equivalent to $\varphi$. Thanks to the axioms (COM) and $(*$ DISTR), the $*-$ introduction rule and propositional reasoning, the satisfaction of such a property amounts to check it in the restricted case of elementary shapes (see Lemma 8).

With the formula $\left|Q_{1}, \ldots, Q_{n}\right\rangle \mid$ we denote a formula of the form either $\left|Q_{1}, \ldots, Q_{n}\right\rangle$ or $\left|Q_{1}, \ldots, \overleftarrow{Q_{i}, \ldots, Q_{n}}\right|$ (this excludes graph formulae of the form $\left.\mid Q_{1}, \ldots, Q_{n}\right]$ ). In the table below, the occurrences of $\left|Q_{1}, \ldots, Q_{n}\right\rangle \mid$ on the left and on the right of every double implication are for the same form, i.e. either both $\left|Q_{1}, \ldots, Q_{n}\right\rangle$ or both $\mid Q_{1}, \ldots, \overleftarrow{Q_{i}, \ldots, Q_{n} \mid}$ (where the position $i$ is the same). Moreover, $0 \leq \beta_{1}<\beta_{1}^{\prime}$ and $0 \leq \beta_{2}<\beta_{2}^{\prime}$. Finally, we write $\varphi_{n}$ to denote

$\left.\varphi_{n} \stackrel{\text { def }}{=}\left(\mid Q_{1} \wedge Q, \ldots, Q_{n}\right] \vee\left|Q_{1} \wedge Q, \ldots, Q_{n}\right\rangle \vee \bigvee_{i \in[1, n]}\left|Q_{1} \wedge Q, \ldots, \overleftarrow{Q_{i}, \ldots, Q_{n}}\right|\right) \wedge$ size $\geq \beta_{1}+\beta_{2}+1$

Derivable formulae about separating conjunctions of elementary shapes

- $\left(\left|Q_{1}, \ldots, Q_{n}\right\rangle \mid \wedge\right.$ size $\left.\geq \beta_{1}\right) *(\mid Q] \wedge$ size $\left.\geq \beta_{2}\right) \Leftrightarrow\left|Q_{1} \wedge Q, \ldots, Q_{n}\right\rangle \mid \wedge$ size $\geq \beta_{1}+\beta_{2}$

- $\left(\left|Q_{1}, \ldots, Q_{n}\right\rangle \wedge\right.$ size $\geq \beta_{1} \wedge \neg$ size $\left.\geq \beta_{1}^{\prime}\right) *(\mid Q] \wedge$ size $\left.\geq \beta_{2}\right)$ $\Leftrightarrow\left|Q_{1} \wedge Q, \ldots, Q_{n}\right\rangle \mid \wedge$ size $\geq \beta_{1}+\beta_{2}$

- $\left(\left|Q_{1}, \ldots, Q_{n}\right\rangle \mid \wedge\right.$ size $\left.\geq \beta_{1}\right) *(\mid Q] \wedge$ size $\geq \beta_{2} \wedge \neg$ size $\left.\geq \beta_{2}^{\prime}\right)$ $\Leftrightarrow\left|Q_{1} \wedge Q, \ldots, Q_{n}\right\rangle \mid \wedge$ size $\geq \beta_{1}+\beta_{2}$

- $\left(\left|Q_{1}, \ldots, Q_{n}\right\rangle \mid \wedge\right.$ size $\geq \beta_{1} \wedge \neg$ size $\left.\geq \beta_{1}^{\prime}\right) *(\mid Q] \wedge$ size $\geq \beta_{2} \wedge \neg$ size $\left.\geq \beta_{2}^{\prime}\right)$ $\Leftrightarrow\left|Q_{1} \wedge Q, \ldots, Q_{n}\right\rangle \mid \wedge$ size $\geq \beta_{1}+\beta_{2} \wedge \neg$ size $\geq\left(\beta_{1}^{\prime}+\beta_{2}^{\prime}\right) \div 1$

- $\left(\mid Q_{1}, \ldots, Q_{n}\right] \wedge$ size $\left.\geq \beta_{1}\right) *(\mid Q] \wedge \neg$ size $\left.\left.\geq 1\right) \Leftrightarrow \mid Q_{1} \wedge Q, \ldots, Q_{n}\right] \wedge$ size $\geq \beta_{1}$

- $\left(\mid Q_{1}, \ldots, Q_{n}\right] \wedge$ size $\geq \beta_{1} \wedge \neg$ size $\left.\geq \beta_{2}\right) *(\mid Q] \wedge \neg$ size $\left.\geq 1\right)$ $\left.\Leftrightarrow \mid Q_{1} \wedge Q, \ldots, Q_{n}\right] \wedge$ size $\geq \beta_{1} \wedge \neg$ size $\geq \beta_{2}$

- $\left(\mid Q_{1}, \ldots, Q_{n}\right] \wedge$ size $\left.\geq \beta_{1}\right) *(\mid Q] \wedge$ size $\left.\geq \beta_{2}+1\right) \Leftrightarrow \varphi_{n}$

- $\left(\mid Q_{1}, \ldots, Q_{n}\right] \wedge$ size $\geq \beta_{1} \wedge \neg$ size $\left.\geq \beta_{1}^{\prime}\right) *(\mid Q] \wedge$ size $\left.\geq \beta_{2}+1\right) \Leftrightarrow \varphi_{n}$

- $\left(\mid Q_{1}, \ldots, Q_{n}\right] \wedge$ size $\left.\geq \beta_{1}\right) *(\mid Q] \wedge$ size $\geq \beta_{2}+1 \wedge \neg$ size $\left.\geq \beta_{2}^{\prime}\right) \Leftrightarrow \varphi_{n}$

- $\left(\mid Q_{1}, \ldots, Q_{n}\right] \wedge$ size $\geq \beta_{1} \wedge \neg$ size $\left.\geq \beta_{1}^{\prime}\right) *(\mid Q] \wedge$ size $\geq \beta_{2}+1 \wedge \neg$ size $\left.\geq \beta_{2}^{\prime}\right) \Leftrightarrow$ $\varphi_{n} \wedge \neg$ size $\geq \beta_{1}^{\prime}+\beta_{2}^{\prime}-1$

Once Lemma 8 is shown, forthcoming Lemma 9 can be easily shown.

Lemma 8. The formulae listed in the table above are derivable in $\mathcal{H}_{c}(*)$ assuming that for any elementary shape $\psi$ of the form either $\mathcal{G} \wedge$ size $\geq \beta$ or $\mathcal{G} \wedge$ size $\geq$ $\beta \wedge \neg$ size $\geq \beta^{\prime}$, we have $\sharp(\mathcal{G}) \leq \beta, \beta<\beta^{\prime}$ and $\forall_{\mathcal{H}_{c}} \neg \psi$.

From Lemmata 7 and 9, we get the main result about $*$-elimination.

Lemma 9. Let $\phi, \psi$ be Boolean combinations of core formulae. There is a Boolean combination of core formulae $\varphi$ such that $\vdash_{\mathcal{H}_{c}(*)}(\phi * \psi) \Leftrightarrow \varphi$.

In the proof of Lemma 9 , if $\vdash_{\mathcal{H}_{c}(*)} \neg \phi$ or $\vdash_{\mathcal{H}_{c}(*)} \neg \psi$, the axiom $(\perp)$ is then used. Otherwise, the proof amounts to prove the statement for elementary shapes 
only, which corresponds to Lemma 8. Let $\mathcal{H M S L}(*, \diamond)$ be the Hilbert-style proof system defined as the union of the axioms and inference rules from $\mathcal{H}_{c}(\diamond)$ and $\mathcal{H}_{c}(*)$ (with the intersection $\mathcal{H}_{c}$ ) augmented with the axiom below:

$$
\left.\left(\mathbf{G}_{30}\right) \quad p \Leftrightarrow(|p\rangle \vee \mid p] \vee|p|\right) \text { with } p \in \text { PROP. }
$$

Theorem 2. $\mathcal{H} M S L(*, \diamond)$ is sound and complete for $M S L(*, \diamond)$.

Proof. (sketch) We need to show that (1) the axiom $\left(\mathbf{G}_{30}\right)$ is valid for $\operatorname{MSL}(*, \diamond)$ (easy), (2) to show that all the axioms and inference rules of $\mathcal{H M S L}(*, \diamond)$ are valid for $\operatorname{MSL}(*, \diamond)$ and $(3)$ to prove that $\vdash_{\mathcal{H M S L}(*, \diamond)} \phi$ for every valid formula $\phi$.

The proof of (2) is a consequence of (1), Lemma 5 and Lemma 7. However, one needs to notice that the validity of the axiom schemas and inference rules can be deduced from the proofs of Lemma 5 and Lemma 7 , even though in $\mathcal{H M S L}(*, \diamond)$, the metavariables $\phi, \psi$ and $\varphi$ used in the axioms and inference rules from $\mathcal{H}_{c}(\diamond)$ and $\mathcal{H}_{c}(*)$, can be safely instantiated by any formula in $\operatorname{MSL}(*, \diamond)$.

The proof of (3) consists in showing that there is a Boolean combination of core formulae $\psi$ such that $\vdash_{\mathcal{H M S L}(*, \diamond)} \phi \Leftrightarrow \psi(\phi$ and $\psi$ are logically equivalent by (2)). For instance, loop from $_{2} 2$ is logically equivalent to $\neg$ size $\geq 2 \wedge \mid \uparrow$, whereas loop $_{2}$ is logically equivalent to $\neg$ size $\geq 3 \wedge|\uparrow, T|$. These equivalences can be derived in $\mathcal{H M S L}(*, \diamond)$. So, $\psi$ is valid and by Theorem 1 , we get $\vdash_{\mathcal{H}_{c}}$ $\psi$ and therefore $\vdash_{\mathcal{H M S L}(*, \diamond)} \psi$. By propositional reasoning, we conclude that $\vdash_{\mathcal{H M S L}(*, \diamond)} \phi$. It remains to prove that $\psi$ exists. The proof is by structural induction using Lemma 6, Lemma 9 and the axiom $\left(\mathbf{G}_{30}\right)$.

\section{Hilbert-style proof system for $\operatorname{MSL}(*,\langle\neq\rangle)$}

In this section, we present a proof system for $\operatorname{MSL}(*,\langle\neq\rangle)$ by using previous developments from $\S 3$ as well as by adapting to infinite models the proof method in [32] for axiomatising the logic of elsewhere $\operatorname{ML}(\langle\neq\rangle)$. The NP upper bound proof for $\operatorname{MSL}(*,\langle\neq\rangle)$ satisfiability in [18] takes advantage of an abstraction accounting only for the number of edges in the model (up to a value depending linearly on the size of the input formula) and whether given a propositional valuation (restricted to the propositional variables occurring in the input formula), there are none, one or two locations satisfying it. The developments below propose a syntactic characterisation for MSL $(*,\langle\neq\rangle)$ validity that also witnesses that the interplay between the number of edges and the constraints on the valuations is very weak. Below, a pure separation formula is understood as a formula in $\operatorname{MSL}(*,\langle\neq\rangle)$ with no occurrences of $\langle\neq\rangle$ and propositional symbols, and a pure modal formula is understood as a formula with no occurrences of $*$ and emp. We denote these families as $\operatorname{MSL}(*)$ and $\operatorname{MSL}(\langle\neq\rangle)$, respectively.

We design the system $\mathcal{H} \operatorname{MSL}(*,\langle\neq\rangle)$ for $\operatorname{MSL}(*,\langle\neq\rangle)$ by the union of the system $\mathcal{H M S L}(\langle\neq\rangle)$ for $\operatorname{MSL}(\langle\neq\rangle)$, of the system $\mathcal{H M S L}(*)$ for $\operatorname{MSL}(*)$, plus the new axioms $(\langle\neq\rangle$ SEP $)$ and $(*$ SEP $)$. 
Axiomatising $\operatorname{ML}(\langle\neq\rangle)$ on MSL models. We introduce $\mathcal{H} \operatorname{MSL}(\langle\neq\rangle)$ for axiomatising the logic $\operatorname{MSL}(\langle\neq\rangle)$, that is designed by augmenting the Hilbert-style system for the logic of elsewhere $\mathrm{ML}(\langle\neq\rangle)$ from [32] by an axiom expressing that $\operatorname{MSL}(\langle\neq\rangle)$ models have an infinite number of locations (namely (INF)). For instance, the formula $\langle\mathrm{U}\rangle(p \wedge[\neq] \neg p) \wedge\langle\mathrm{U}\rangle(\neg p \wedge[\neq] p)$, where $[\neq] \phi \stackrel{\text { def }}{=} \neg\langle\neq\rangle \neg \phi$ and $\langle\mathrm{U}\rangle \phi \stackrel{\text { def }}{=} \phi \vee\langle\neq\rangle \phi$, is satisfiable in some $\operatorname{ML}(\langle\neq\rangle)$ model with two locations exactly whereas it is unsatisfiable for $\operatorname{MSL}(\langle\neq\rangle)$. As usual, the axiom schemas and modus ponens for propositional calculus are part of $\mathcal{H M S L}(\langle\neq\rangle)$.

Axioms and inference rule for $\mathcal{H M S L}(\langle\neq\rangle)$

(K) $[\neq](\phi \Rightarrow \psi) \Rightarrow([\neq] \phi \Rightarrow[\neq] \psi)$
(B) $\phi \Rightarrow[\neq]\langle\neq\rangle \phi$$\quad$ (ALIO) $\phi \Rightarrow([\neq] \phi \Rightarrow[\neq][\neq] \phi)$

(INF) $\bigvee_{X \subseteq\left\{p_{1}, \ldots, p_{n}\right\}}\langle\mathrm{U}\rangle\left(\psi_{X} \wedge\langle\neq\rangle \psi_{X}\right)$ for every $\left\{p_{1}, \ldots, p_{n}\right\} \subset_{\text {fin }}$ PROP, where $\bar{\psi}_{X}$ stands for $\left(\bigwedge_{p \in X} p\right) \wedge\left(\bigwedge_{p \in\left(\left\{p_{1}, \ldots, p_{n}\right\} \backslash X\right)} \neg p\right)$.

Necessitation rule: $\frac{\phi}{[\neq] \phi}$

In $\mathcal{H M S L}(\langle\neq\rangle)$, the axiom $(\mathbf{K})$ and the necessitation rule are standard for normal modal logics, whereas the axiom (B) (resp. (ALIO)) takes care of the symmetry (resp. the aliotransitivity) of the difference relation. As the MSL $(\langle\neq\rangle)$ models are necessarily infinite (by contrast to the models for the logic of elsewhere), we add the axiom (INF).

Lemma 10. Axioms and rules in $\mathcal{H} M S L(\langle\neq\rangle)$ are valid for $M S L(\langle\neq\rangle)$.

An $\operatorname{MSL}(\langle\neq\rangle)$ model $\mathfrak{M}=\langle\mathbb{N}, \mathfrak{R}, \mathfrak{V}\rangle$ can be understood as the $\operatorname{ML}(\langle\neq\rangle)$ model $\langle\mathbb{N}, \neq, \mathfrak{V}\rangle$ since the language MSL $(\langle\neq\rangle)$ does not require to use of $\mathfrak{R}$ to evaluate formulae. So, in the sequel, we assume that the models for $\operatorname{ML}(\langle\neq\rangle)$ are of the form $\langle\mathfrak{W}, \neq, \mathfrak{V}\rangle$, whereas those for $\operatorname{MSL}(\langle\neq\rangle)$ are the restrictions with $\mathfrak{W}=\mathbb{N}$.

Lemma 11. $\mathcal{H} M S L(\langle\neq\rangle)$ is sound and complete for $M S L(\langle\neq\rangle)$.

The completeness of $\mathcal{H M S L}(\langle\neq\rangle)$ is shown by adapting the completeness proof from [32] and by taking advantage of the infinity axiom (INF).

Axiomatising $\operatorname{MSL}(*)$. We present the Hilbert-style system $\mathcal{H M S L}(*)$ for the $\operatorname{logic} \operatorname{MSL}(*)$. It is designed as a fragment of the Hilbert-style system $\mathcal{H M S L}(*, \diamond)$ from $\S 3$ by simplifying the axioms and by keeping only what is needed for MSL $(*)$. Axioms and inference rules for $\mathcal{H} \operatorname{MSL}(*)$

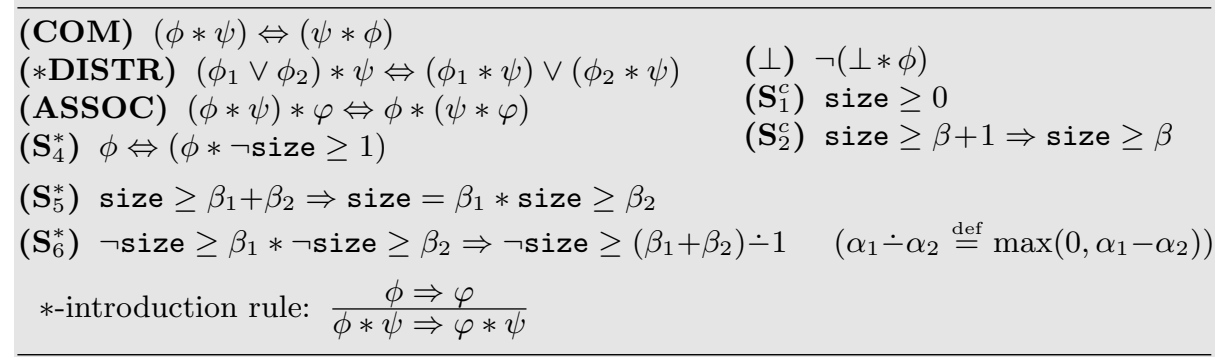


As $\operatorname{MSL}(*)$ is a fragment of both $\operatorname{MSL}(*, \diamond)$ and $\operatorname{MSL}(*,\langle\neq\rangle)$, it should not come as a surprise that all the axioms above were already introduced in $\S 3$. Before proving completeness, we establish a few results about $\mathcal{H} \mathrm{MSL}(*)$ that can be shown along the lines of $\S 3$ but drastic simplifications apply.

Lemma 12. Axioms and rules in $\mathcal{H} M S L(*)$ are valid for $M S L(*)$.

This is a consequence of the correctness for $\mathcal{H M S L}(*, \diamond)$ (see $\S 3)$, as derivability in $\mathcal{H M S L}(*)$ implies derivability in $\mathcal{H M S L}(*, \diamond)$.

Lemma 13. Given $\phi$ in $M S L(*), \vdash_{\mathcal{H} M S L(*)} \phi \Leftrightarrow \psi$ for some size formula $\psi$.

Proving completeness is now by an easy verification.

Lemma 14. $\mathcal{H} M S L(*)$ is sound and complete for $M S L(*)$.

Proof. (sketch) Soundness is from Lemma 12. It remains to establish completeness. Let $\phi$ be a formula that is valid for $\operatorname{MSL}(*)$. First, notice that the following property holds: if $\vdash_{\mathcal{H M S L}(*)} \phi \Leftrightarrow \phi^{\prime}$, then $\vdash_{\mathcal{H M S L}(*)} \psi[\phi]_{\rho} \Leftrightarrow \psi\left[\phi^{\prime}\right]_{\rho}$, where the formula $\psi[\phi]_{\rho}$ stands for the formula obtained from $\psi$ by replacing the formula at the occurrence $\rho$ by the formula $\phi$.

By Lemma 13, it is easy to show that there is a size formula $\phi^{\prime}$ in CNF such that $\vdash_{\mathcal{H M S L}(*)} \phi \Leftrightarrow \phi^{\prime}$ in $\mathcal{H} \operatorname{MSL}(*)$ and each conjunct of $\phi^{\prime}$ contains at most 2 size literals, and they are of distinct polarity. By Lemma 12, $\phi^{\prime}$ is also $\operatorname{MSL}(*)$ valid and therefore each conjunct is valid. If a conjunct is of the form size $\geq \beta$, then $\beta=0$ as size $\geq \beta$ should be valid. As size $\geq 0=\top$, we have $\vdash_{\mathcal{H M S L}(*)}$ size $\geq 0$. No conjunct can be of the form $\neg($ size $\geq \beta)$ as no formula of the form $\neg($ size $\geq \beta)$ is valid. If a conjunct is of the form size $\geq \beta \vee \neg\left(\right.$ size $\left.\geq \beta^{\prime}\right)$, then $\beta^{\prime} \geq \beta$ as size $\geq \beta \vee \neg\left(\right.$ size $\left.\geq \beta^{\prime}\right)$ is required to be valid. By propositional reasoning and by using $\left(\beta^{\prime}-\beta\right)$ times the axiom $\left(\mathbf{S}_{2}^{c}\right)$, we can conclude that $\vdash_{\mathcal{H M S L}(*)}\left(\operatorname{size} \geq \beta^{\prime}\right) \Rightarrow($ size $\geq \beta)$ and therefore $\vdash_{\mathcal{H M S L}(*)}$ size $\geq \beta \vee \neg\left(\right.$ size $\left.\geq \beta^{\prime}\right)$ by propositional reasoning. Hence, $\vdash_{\mathcal{H M S L}(*)} \phi^{\prime}$, and since $\vdash_{\mathcal{H M S L}(*)} \phi \Leftrightarrow \phi^{\prime}$, by propositional reasoning, we also get $\vdash_{\mathcal{H} \operatorname{MSL}(*)} \phi$.

Putting all together: axiomatising $\operatorname{MSL}(*,\langle\neq\rangle)$. It is now time to define the Hilbert-style proof system $\mathcal{H M S L}(*,\langle\neq\rangle)$ obtained from the calculus containing the axioms and rules from $\mathcal{H M S L}(*)$ and $\mathcal{H M S L}(\langle\neq\rangle)$. We need however to introduce two more axioms, stating that pure separation formulae can be separated from pure modal formulae. Notice that this property has some similarities with the separation theorem for Past LTL from [23].

Separation axioms

$(\langle\neq\rangle$ SEP $)\langle\neq\rangle(\phi \wedge \psi) \Leftrightarrow(\langle\neq\rangle \phi) \wedge \psi$ where $\psi$ is a pure separation formula $(* \mathbf{S E P}) \phi *\left(\phi^{\prime} \wedge \psi\right) \Leftrightarrow\left(\phi * \phi^{\prime}\right) \wedge \psi$ where $\psi$ is a pure modal formula

Lemma 15. Axioms and rules in $\mathcal{H} M S L(*,\langle\neq\rangle)$ are valid for $M S L(*,\langle\neq\rangle)$.

Completeness of $\mathcal{H M S L}(*,\langle\neq\rangle)$ takes advantage of the resp. completeness of $\mathcal{H} \operatorname{MSL}(\langle\neq\rangle)$ and $\mathcal{H M S L}(*)$, and the fact that for all pure modal (resp. separation) formulae $\phi_{M}$ (resp. $\left.\phi_{S}\right), \phi_{M} \vee \phi_{S}$ is valid iff $\phi_{M}$ is valid or $\phi_{S}$ is valid. 
Theorem 3. $\mathcal{H} M S L(*,\langle\neq\rangle)$ is sound and complete for $M S L(*,\langle\neq\rangle)$.

Proof. (sketch) Soundness is from Lemma 15. Let us establish completeness. Let $\phi$ be valid for $\operatorname{MSL}(*,\langle\neq\rangle)$. By using the axioms $(\langle\neq\rangle \mathbf{S E P})$ and $(* \mathbf{S E P})$, one can show that there is a formula $\phi^{\prime}$ such that $\vdash_{\mathcal{H M S L}(*,\langle\neq\rangle)} \phi \Leftrightarrow \phi^{\prime}$ and $\phi^{\prime}$ is a Boolean combination of formulae from $\operatorname{MSL}(*) \cup \operatorname{MSL}(\langle\neq\rangle)$. By the validity of the axioms and inference rules (Lemma 15), we have that $\phi^{\prime}$ is $\operatorname{MSL}(*,\langle\neq\rangle)$ valid as well. By propositional reasoning in $\mathcal{H M S L}(*,\langle\neq\rangle)$, there is $\phi^{\prime \prime}$ in CNF such that $\vdash_{\mathcal{H} \text { MSL }(*,\langle\neq\rangle)} \phi^{\prime} \Leftrightarrow \phi^{\prime \prime}$ and $\phi^{\prime \prime}$ is a conjunction of disjunctions of the form $\phi_{M} \vee \phi_{S}$ where $\phi_{M}$ is a pure modal formula and $\phi_{S}$ is a pure separation formula. Again, by the validity of the axioms and inference rules, each disjunction $\phi_{M} \vee \phi_{S}$ is valid in $\operatorname{MSL}(*,\langle\neq\rangle)$.

Now, one can show that $\phi_{M} \vee \phi_{S}$ is valid iff $\phi_{M}$ is valid for $\operatorname{MSL}(\langle\neq\rangle)$ or $\phi_{S}$ is valid for $\operatorname{MSL}(*)$. By completeness of $\mathcal{H M S L}(\langle\neq\rangle)$ and $\mathcal{H} \mathrm{MSL}(*)$, we get that $\phi_{M} \vee \phi_{S}$ is valid iff $\vdash_{\mathcal{H M S L}(\langle\neq\rangle)} \phi_{M}$ or $\vdash_{\mathcal{H M S L}(*)} \phi_{S}$. This is sufficient to conclude that $\vdash_{\mathcal{H} \text { MSL }(*,\{\neq\rangle)} \phi_{M} \vee \phi_{S}$. Consequently, for each disjunct $\phi_{M} \vee \phi_{S}$ of $\phi^{\prime \prime}$, we have $\vdash_{\mathcal{H M S L}(*,\langle\neq\rangle)} \phi_{M} \vee \phi_{S}$ and therefore by propositional reasoning, we get that $\vdash_{\mathcal{H M S L}(*,\langle\neq\rangle)} \phi^{\prime \prime}$. As $\vdash_{\mathcal{H M S L}(*,\langle\neq\rangle)} \phi \Leftrightarrow \phi^{\prime}$ and $\vdash_{\mathcal{H M S L}(*,\langle\neq\rangle)} \phi^{\prime} \Leftrightarrow \phi^{\prime \prime}$, we get that $\vdash_{\mathcal{H} \operatorname{MSL}(*,\langle\neq\rangle)} \phi$. Therefore, $\mathcal{H M S L}(*,\langle\neq\rangle)$ is complete.

\section{Concluding remarks}

We provided an axiomatisation for the $\operatorname{logics} \operatorname{MSL}(*, \diamond)$ and $\operatorname{MSL}(*,\langle\neq\rangle)$, despite the well-known difficulties to axiomatise logics equipped with operators that update the models in the evaluation process. Such operators are ubiquitous in theoretical computer science and in knowledge representation areas, and we hope that our calculi shed some new light on their expressive power. For the axiomatisation of $\operatorname{MSL}(*, \diamond)$ we had to identify the core properties that can be expressed in the logic, partially following the semantical analysis from [18]. We also had to express them in the language with the so-called core formulae. Implicitly, the axiomatisation is divided into two parts: axioms and rules to transform any formula of $\operatorname{MSL}(*, \diamond)$ into a Boolean combination of core formulae and the axiomatisation of these Boolean combinations. For the axiomatisation of $\operatorname{MSL}(*,\langle\neq\rangle)$, we use a similar approach, except that we had to adapt the axiomatisation of the logic of elsewhere from [32] to infinite models and to implement syntactically a separation principle satisfied by $\operatorname{MSL}(*,\langle\neq\rangle)$. It is worth noting that the completeness of $\mathcal{H} \operatorname{MSL}(*, \diamond)$ and $\mathcal{H M S L}(*,\langle\neq\rangle)$ does not imply their strong completeness, as $\operatorname{MSL}(*)$ is not compact. Let us consider $X_{\infty}=\{$ size $\geq \beta \mid \beta \in \mathbb{N}\}$. Indeed, for both logics, $X_{\infty}$ is unsatisfiable, since MSL models have finite accessibility relations. Strong completeness would imply that $\perp$ could be derived from $X_{\infty}$. As all rules are finitary, then there is a finite subset $X \subseteq X_{\infty}$ such that $X \vdash \perp$, or equivalently $\vdash \bigvee_{\psi \in X} \neg \psi$. This leads to a contradiction by the correctness of $\mathcal{H M S L}(*, \diamond)$ and $\mathcal{H} \operatorname{MSL}(*,\langle\neq\rangle)$. The same argument can be used for other finitary proof systems, with the same set $X_{\infty}$.

As part of future work, we aim at Hilbert-style axiomatisations for separation logics having a notion of core formulae (see e.g. $[22,19]$ ), or for very expressive 
modal separation logics such as $\operatorname{MSL}(*,\langle\neq\rangle, \diamond)$. Additionally, the expressivity characterisation provided by core formulae appears to be handy not only as the basic ingredient for the axiomatisations, but also for studying other problems, such as the implementation of proof methods, or the analysis of meta-theoretical properties of the logics.

Ackowledgements. This work was partially supported by ANPCyT-PICTs-2017-1130 and 2016-0215, MinCyT Córdoba, SeCyT-UNC, the Laboratoire International Associé INFINIS and the Centre National de la Recherche Scientifique (CNRS).

\section{References}

1. C. Areces and R. Fervari. Hilbert-style axiomatization for hybrid XPath with data. In JELIA'16, volume 10021 of LNCS, pages 34-48. Springer, 2016.

2. C. Areces, R. Fervari, and G. Hoffmann. Tableaux for relation-changing modal logics. In FroCos'13, volume 8152 of LNCS, pages 263-278, 2013.

3. C. Areces, R. Fervari, and G. Hoffmann. Relation-changing modal operators. Logic Journal of the IGPL, 23(4):601-627, 2015.

4. G. Aucher, J. van Benthem, and D. Grossi. Modal logics of sabotage revisited. $J L C, 28(2): 269-303,2018$.

5. Ph. Balbiani and T. Tinchev. Unification in modal logic Alt 1 . In AiML'16, pages 117-134. College Publications, 2016.

6. J. Berdine, C. Calcagno, and P. O'Hearn. A decidable fragment of separation logic. In FSTÉTCS'04, volume 3328 of $L N C S$, pages 97-109. Springer, 2004.

7. P. Blackburn, M. de Rijke, and Y. Venema. Modal Logic. Cambridge University Press, 2001

8. P. Blackburn, J.F. van Benthem, and F. Wolter, editors. Handbook of Modal Logic. Elsevier, 2006.

9. J. Boudou. Decidable logics with associative binary modalities. In $C S L$ '17, volume 82 of LIPIcs, pages 1-15. Schloss Dagstuhl - Leibniz-Zentrum fuer Informatik, 2017.

10. R. Brochenin, S. Demri, and E. Lozes. Reasoning about sequences of memory states. Annals of Pure and Applied Logic, 161(3):305-323, 2009.

11. J. Brotherston and J. Villard. Parametric completeness for separation theories. In POPL'14, pages 453-464. ACM, 2014.

12. D. Calvanese, T. Kotek, M. Simkus, H. Veith, and F. Zuleger. Shape and content - A database-theoretic perspective on the analysis of data structures. In IFM'14 volume 8739 of $L N C S$, pages 3-17. Springer, 2014.

13. J.-R. Courtault and D. Galmiche. A modal separation logic for resource dynamics. $J L C, 28(4): 733-778,2018$.

14. J.-R. Courtault, H. van Ditmarsch, and D. Galmiche. A public announcement separation logic. Mathematical Structures in Computer Science. To appear.

15. J.-R. Courtault, H. van Ditmarsch, and D. Galmiche. An epistemic separation logic. In WoLLIC'15, volume 9160 of $L N C S$, pages 156-173. Springer, 2015.

16. A. Dawar, Ph. Gardner, and G. Ghelli. Expressiveness and complexity of graph logic. IC, 205(3):263-310, 2007.

17. S. Demri and M. Deters. Two-variable separation logic and its inner circle. ToCL, 2(16), 2015

18. S. Demri and R. Fervari. On the complexity of modal separation logics. In $A i M L$ '18, pages 179-198. College Publications, 2018. 
19. S. Demri, É. Lozes, and A. Mansutti. The effects of adding reachability predicates in propositional separation logic. In FoSSaCS, volume 10803 of $L N C S$, pages 476-493. Springer, 2018.

20. A. Doumane. Constructive completeness for the linear-time $\mu$-calculus. In LICS'17, pages 1-12. IEEE Computer Society, 2017.

21. H.D. Ebbinghaus and J. Flum. Finite Model Theory. Perspectives in Mathematical Logic. Springer Berlin Heidelberg, 1999.

22. M. Echenim, R. Iosif, and N. Peltier. On the expressive completeness of Bernays-Schönfinkel-Ramsey separation logic. Technical Report arXiv:1802.00195, arXiv:cs.LO, February 2018. To appear in FOSSACS'19.

23. D. Gabbay. The declarative past and imperative future. In Temporal Logic in Specification, Altrincham, UK, volume 398 of LNCS, pages 409-448. Springer, 1987.

24. N. Gierasimczuk, L. Kurzen, and F.R. Velázquez-Quesada. Learning and teaching as a game: A sabotage approach. In LORI'09, volume 5834 of $L N C S$, pages 119-132. Springer, 2009.

25. A. Herzig. A simple separation logic. In WoLLIC'13, volume 8071 of $L N C S$, pages 168-178. Springer, 2013.

26. C.A.R. Hoare. An axiomatic basis for computer programming. Communications of the ACM, 12(10):576-580, 1969.

27. Z. Hou, R. Clouston, R. Goré, and A. Tiu. Modular labelled sequent calculi for abstract separation logics. ToCL, 19(2):13:1-13:35, 2018.

28. X. Lu, C. Tian, and Z. Duan. Temporalising separation logic for planning with search control knowledge. In IJCAI'17, pages 1167-1173, 2017.

29. D. Pym, J. Spring, and P.W. O'Hearn. Why separation logic works. Philosophy \& Technology, pages 1-34, 2018.

30. J.C. Reynolds. Separation logic: a logic for shared mutable data structures. In LICS'02, pages 55-74. IEEE, 2002.

31. S. Schmitz. Complexity hierarchies beyond Elementary. ACM Transactions on Computation Theory, 8(1):3:1-3:36, 2016.

32. K. Segerberg. A note on the logic of elsewhere. Theoria, 47:183-187, 1981.

33. Y. Shoham and K. Leyton-Brown. Multiagent Systems: Algorithmic, GameTheoretic, and Logical Foundations. Cambridge University Press, New York, NY, USA, 2008.

34. J. van Benthem. An essay on sabotage and obstruction. In Mechanizing Mathematical Reasoning, Essays in Honor of Jörg Siekmann on the Occasion of his 69th Birthday, pages 268-276. Springer Verlag, 2005.

35. J. van Benthem. Logical Dynamics of Information and Interaction. Cambridge University Press, 2011.

36. H. van Ditmarsch, W. van der Hoek, and B. Kooi. Dynamic Epistemic Logic, volume 337 of Synthese Library Series. Springer, Dordrecht, 2008.

37. Y. Wang and Q. Cao. On axiomatizations of public announcement logic. Synthese, 190(Supplement-1):103-134, 2013. 